\title{
Endorsement of trial registration and the CONSORT statement by the Revista Brasileira de Fisioterapia
}

\section{Adesão ao registro de ensaios clínicos e às recomendações do CONSORT pela Revista Brasileira de Fisioterapia}

$\mathrm{T}$ he Revista Brasileira de Fisioterapia/Brazilian Journal of Physical Therapy (RBF/BJPT) has taken an important step by implementing editorial policies that are relevant to the publication of randomized controlled trials.

The first policy implemented is that authors of clinical trials need to prospectively register their trials, if the trial is to be published in the journal. The principal rationale for trial registration is to avoid selective reporting and publication bias ${ }^{1}$. The problem is that trials that report large and positive effects of the intervention being evaluated are more likely to be published ${ }^{2}$; and this selective reporting may create overly optimistic impressions of the effects of treatment.

Trial registration is an ethical requirement of research that has been endorsed by the World Medical Association: "Every clinical trial must be registered in a publicly accessible database before the recruitment of the first subject (paragraph 19)"3. Trial registration is also mandated in the 2008 revision of the Declaration of Helskinki. Registering a trial is very easy. Authors can visit the website of one of the clinical trials registries endorsed by the International Committee of Medical Journal Editors (ICJME), such as the Australian New Zealand Clinical Trials Registry (http:// www.anzctr.org.au/) or the American Clinical Trials Registry (www.clinicaltrials.gov). The websites provide simple and useful information on the planned conduct of the trial, including a description of the interventions being evaluated, a statement of the study hypotheses, definition of all primary and secondary outcomes and eligibility criteria. From 2007 clinical trials are only considered for publication in the RBF if they have been prospectively registered in an ICMJE-endorsed clinical trial registry.

The second editorial policy is to endorse the recommendations of the Consolidated Standards of Reporting Trials (CONSORT) statement. The CONSORT statement provides recommendations for authors regarding how to prepare reports of trial findings; facilitating complete and transparent reporting, and aiding critical appraisal and interpretation. The CONSORT statement was first developed in $1996^{4}$ by an international group of clinical trialists, statisticians, epidemiologists and biomedical editors, and was then revised in $2001^{5}$. It has been supported by a large number of medical journals and editorial groups, such as the ICMJE and the World Association of Medical Editors. To date, the CONSORT statement has been translated into 10 languages, including Portuguese. The full version can be downloaded free of charge at the following URL: http://www.consort-statement.org/consort-statement/.

The CONSORT statement comprises a 22-item checklist and a flow diagram, along with explanations and examples for each item. The items focus on how the trial was designed, analysed and interpreted; while the flow diagram presents the progress of participants through the trial. There are also extensions of the CONSORT statement to cover issues relevant to specific types of trials, trials evaluating non-pharmacological interventions ${ }^{6.7}$, for example. 
The endorsement of the CONSORT statement by the RBF will bring many advantages for authors, editors/reviewers and readers of the RBF. Firstly the CONSORT statement will greatly assist authors who are submitting clinical trial manuscripts for publication, as the CONSORT statement outlines all the information that should be included in the manuscript in an easy, reliable and transparent structure. Secondly editors and reviewers from RBF will be able to check the completeness of the trial reporting, so the CONSORT statement will help reviewers to provide better feedback to authors. Finally, RBF readers will be able to read clearer articles, which will enable them to more readily interpret the results of the trials and decide if particular clinical trials provide valid and interpretable information that will help them select the optimal treatment for their patients 8 .

\section{References $: \because$.}

1. De Angelis C, Drazen JM, Frizelle FA, Haug C, Holy J, Horton R, et al. Clinical trial registration: a statement from the International Committee of Medical Journal Editors. N Engl J Med. 2004;351:1250-1.

2. Hopewell S, Loudon K, Clarke MJ, Oxman AD, Dickersin K. Publication bias in clinical trials due to statistical significance or direction of trial results. Cochrane Database Syst Rev. 2009;MR000006.

3. World Medical Association Declaration of Helsinki. Ethical principles for medical research involving human subjects: World Medical Association Declaration of Helsinki. 2008:1-5.

4. Begg C, Cho M, Eastwood S, Horton R, Moher D, Olkin I, et al. Improving the quality of reporting of randomized controlled trials. The CONSORT statement. JAMA. 1996;276(8):637-9.

5. Altman DG, Schulz KF, Moher D, Egger M, Davidoff F, Elbourne D, et al. The revised CONSORT statement for reporting randomized trials: explanation and elaboration. Ann Intern Med. 2001;134(8):663-94.

6. Boutron I, Moher D, Altman DG, Schulz KF, Ravaud P; CONSORT Group. Extending the CONSORT statement to randomized trials of nonpharmacologic treatment: explanation and elaboration. Ann Intern Med. 2008;148(4):295-309.

7. Boutron I, Moher D, Altman DG, Schulz KF, Ravaud P; CONSORT Group. Methods and processes of the CONSORT Group: example of an extension for trials assessing nonpharmacologic treatments. Ann Intern Med. 2008;148(4):W60-6.

8. Vaarbakken K, Ljunggren AE, Hendricks EJ. The importance of the new CONSORT Statement for clinicians. Aust J Physiother. 2008;54(3):155-6.

Leonardo O. P. Costa ${ }^{1,3}$, Christopher G. Maher ${ }^{1}$, Anne M. Moseley ${ }^{1}$, Catherine Sherrington ${ }^{1}$, Robert D. Herbert ${ }^{1}$, Mark R. Elkins ${ }^{2}$

${ }^{1}$ Musculoskeletal Division, The George Institute for International Health, Sydney, Australia

${ }_{2}^{2}$ Department of Respiratory Medicine, Royal Prince Alfred Hospital, Australia

${ }^{3}$ Masters in Physical Therapy Program, Universidade da Cidade de São Paulo (UNICID), São Paulo (SP), Brazil 